Research Article

SPECIAL ISSUE

with invited papers from the $47^{\text {th }}$ International Symposium „Actual tasks on Agricultural Engineering“ (ATAE), $5^{\text {th }}-7^{\text {th }}$ March 2019, Opatija, Croatia, http://atae.agr.hr/, Editor: Prof. Andreas Gronauer

\title{
Conflicts and resolutions in managing water allocation at the watershed scale
}

\section{Konflikte und Lösungen beim Management der Wasserzuteilung auf der Ebene des Wassereinzugsgebietes}

\author{
Zorica Srdjevic $^{1 *}$, Bojan Srdjevic ${ }^{1}$, Paulo Melo ${ }^{2}$, Luísa Jorge ${ }^{3}$
}

\author{
${ }^{1}$ Faculty of Agriculture, University of Novi Sad, Trg D.Obradovica 8, Novi Sad, Serbia \\ ${ }^{2}$ Centre for Business and Economics Research, University of Coimbra and INESC Coimbra, Dias da Silva 165, 3004-512 Coimbra, Portugal \\ ${ }^{3}$ Polytechnic Institute of Bragança, Campus de Santa Apolónia, 5300-253 Bragança, Portugal \\ * Corresponding author: srdjevicz@polj.uns.ac.rs
}

Received: 14 May 2019, received in revised form: 3 August 2019, accepted: 4 August 2019

\begin{abstract}
Summary
Multiple runs of a river basin model produced information about water allocation under different users' priorities, creating a set of allocation scenarios as possible decision alternatives. To identify the most desired scenario that will, expectedly, be more readily accepted and implemented, involvement of stakeholders and reaching the consensus among them in evaluating scenarios are essential. This article describes methodology for integrating multi-criteria optimization as an efficient tool for the evaluation of scenarios in a group context, with river basin simulation-optimization models. Methodology was developed within the scope of the bilateral project Serbia-Portugal, and it consisted of five phases: defining the preference schemes of allocation, running the ACQUANET model, evaluating the criteria and strategies with analytic hierarchy process, aggregation and initial search for consensus in subgroups, and obtaining the final consensus converged result (best management strategy). The approach was tested on the water allocation problem in the Nadela watershed in Vojvodina Province in Serbia, with participation of 23 stakeholders. Promising results recommended the approach for the testing in different conditions in the area near Bragança in northeast Portugal (Sabor watershed).
\end{abstract}

Keywords: river basin model, allocation scenarios, stakeholders, multi-criteria optimization, consensus

\section{Zusammenfassung}

Mehrere Durchläufe eines Flußbeckenmodels generierten Informationen über die Wasserzuteilung bei verschiedenen Nutzerprioritäten, dabei wurde ein Satz an Zuteilungsszenarien für mögliche Entscheidungsalternativen erstellt. Um das am meisten erwünschte Szenario zu identifizieren, das erwartungsgemäß leichter akzeptiert und umgesetzt wird, ist die Einbeziehung von Stakeholdern und das Erreichen eines Konsenses zwischen ihnen bei der Bewertung von Szenarien essenziell. Der Artikel beschreibt die Methodik zur Integration der Mehrkriterienoptimierung als effizientes Werkzeug zur Bewertung von Szenarien im Gruppenkontext mit Simulationsoptimierungsmodellen für Flußbecken. Die Methodik wurde im Rahmen des bilateralen Projekts zwischen Serbien und Portugal entwickelt und bestand aus fünf Phasen: Festlegung der bevorzugten Zuteilungsschemata, Laufenlassen des ACQUANET-Modells, Bewertung von Kriterien und Strategien mit analytischem Hierarchieprozess, Aggregation und anfängliche Suche nach Konsens in Untergruppen und Erzielung des endgültigen konsenskonvergenten Ergebnisses (beste Managementstrategie). Der Ansatz wurde in Bezug auf das Wasserverteilungsproblem im Wassereinzugsgebiet Nadela in der Provinz Vojvodina in Serbien getestet, unter Beteiligung von 23 Stakeholdern. Vielversprechende Ergebnisse empfehlen den Ansatz für die Erprobung unter verschiedenen Bedingungen in der Nähe von Bragança im Nordosten Portugals (Wassereinzugsgebiet Sabor).

Schlagworte: Flußgebietsmodell, Zuteilungsszenarien, Interessengruppen, Multi-Kriterien-Optimierung, Konsens 


\section{Introduction}

The research results presented in this article were created under the bilateral project "Multi-criteria decision making/Analytic hierarchy process applications in solving problems of priority water allocation for agricultural users" (2018-2019). This project is being developed by the University of Novi Sad (Serbia) and the University of Coimbra (Portugal). As a recommendation, in line with the EU Water Framework Directive, the research team suggests that exploitation of water resources in watersheds in Serbia and Portugal should be planed having in mind the existence of different stakeholders, their possible conflicts, and also local economic and political environments. There are specifics and priorities in water management for each country; in the case study area selected within the bilateral project in Serbia, water allocation conflicts are most common among agriculture, industry, and other purposes (outdoor activities, tourism). In Portuguese case study, conflict exists among agriculture, tourism, and energy production. What is common to both countries is that planning should be supported by adequate tools in order to realistically determine how much water is available, to whom and when, and to identify management strategies acceptable to most stakeholders in watersheds of the two countries.

Current developments in water resources management and agriculture in general are characterized by the use of several multi-criteria decision-making methods and tools, advanced information technologies, and participation of the involved stakeholders from different sectors. In spite of significant IT sector development in the past two decades, it is clear that the use of expert systems, simulation and optimization models, and supporting tools for decision makers (DMs) does not yet reach an adequate level. In addition, the communication of issues between the scientific community and society at large still lacks the desired transparency. To take into account different interest of stakeholders, complexities, uncertainties, conflicts, equity, and sustainability issues in water resources management and agriculture, participative forms of decision-making frameworks are necessary. The article proposes such framework through integration of multi-criteria optimization tools (analytic hierarchy process and consensus convergence model) with river basin simulation-optimization model (ACQUANET) in a way that water allocation conflicts can be better recognized, modeled, and solved.

The methodology consist of the following steps: (a) applying river basin simulation-optimization model to determine multiyear water allocation to different water users for several priority schemes of water distribution; (b) structuring the decision-making problem as a hierarchy and applying the analytic hierarchy process (AHP) (Saaty, 1980) by stakeholders (DMs); and (c) grouping stakeholders into subgroups and performing aggregation of computed priorities by the consensus convergence model (Lehrer and Wagner, 1981), first within each subgroup and then between subgroups. The final consensus-based solution is declared as the final group decision.

The Nadela watershed, a typical regional hydro system in Serbia, is selected as a first case study example (the second system will be selected from Portugal) to demonstrate the application of the methodology on resolving major conflicts between upstream and downstream users of the system.

The Nadela watershed is located in the south-east area of Vojvodina Province in Serbia (Figure 1). There are different water uses of this hydro system. Main purposes of the system are drainage, industrial water supply, collecting used waters, irrigation, and other purposes (Srdjevic and Srdjevic, 2019; Srdjevic et al., 2019). The main concerns in the watershed are that along the first $30 \mathrm{~km}$ of the Nadela canal, water is of the desired quality ("blue and clean") and mostly used for irrigation; however, during the summer season and along the downstream final $15 \mathrm{~km}$ (before canal's confluence to the Danube), it is not always possible to support the ecological minimum flow of $0.5 \mathrm{~m}^{3} \mathrm{~s}^{-1}$. In consequence, there is high, uncontrolled, pollution of this canal. The problem to be solved is thus stated as to identify a water allocation strategy that will ensure well-balanced system use and long-term satisfaction of prescribed system purposes and users' expectations. This strategy has to respect system capacity and its technical characteristics and but also interests of the society, including sharing of benefits, environmental protection, and balanced economic development of municipalities.

\section{Materials and methods}

The basic problems for water allocation in any watershed (river basin) can be stated as follows:

(a) Water flow is seasonally fluctuating, leading to quantity problems throughout the watershed;

(b) In some parts of the watershed, water quality cannot achieve a satisfactory level;

(c) Usually, there is a gap between financial demands and actual investment; existing sources of financing available at most water management levels and in 


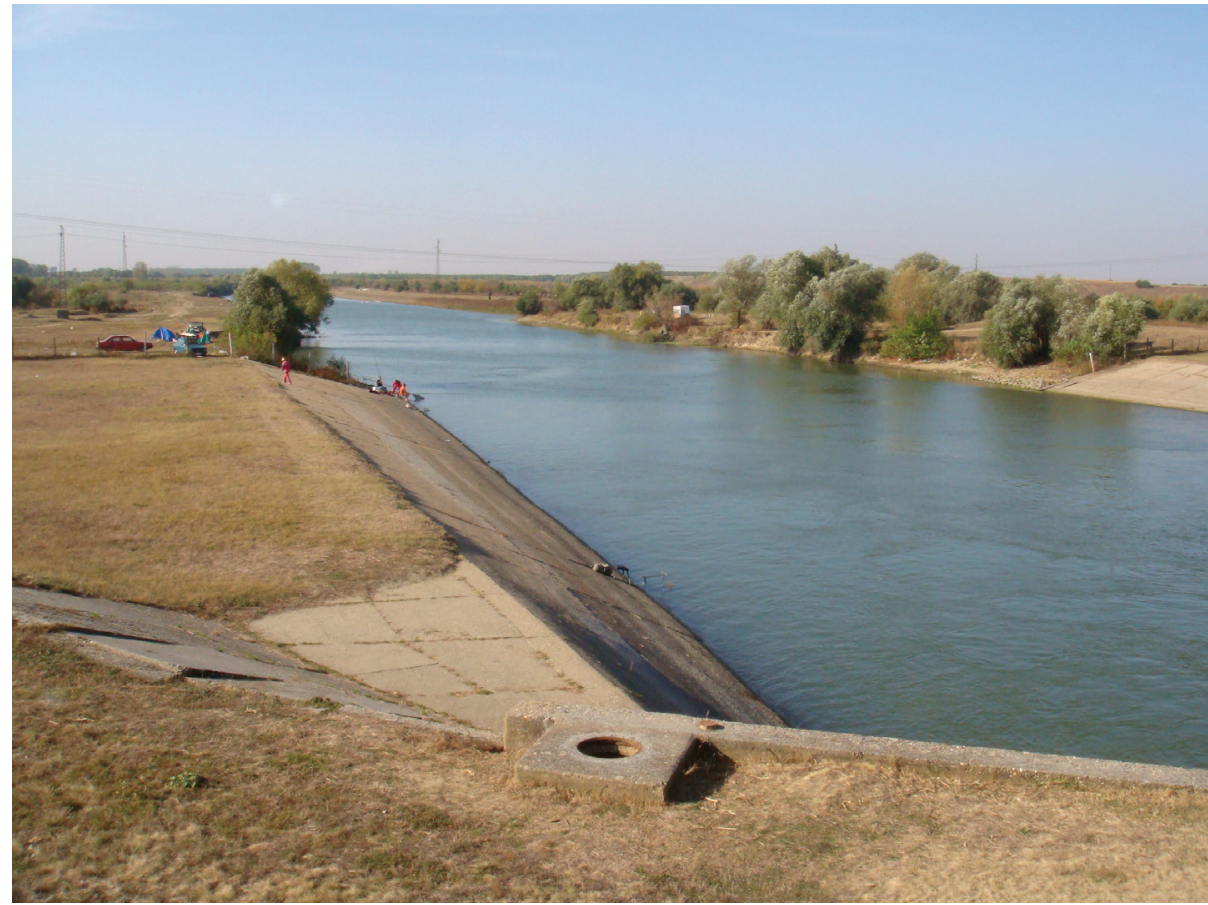

Figure 1. Regional hydro system Nadela

Abbildung 1. Das regionale Wassersystem Nadela

particular segments could be several times less than those required;

(d) Management of the water resources system could be inadequate (inefficient);

(e) Public participation is not encouraged, and sometimes not even possible.

A main strategic goal in the water sector is to enable maintenance and development of a water regime that attempts to achieve the best and most complete engineering, financial, and ecological solutions, accounting for integrated water management, water protection, protection against harmful effects of water, and water use. To accomplish this goal, contemporary road maps suggest that an integral, complex, and unique water resources development system should be created. Creating this system requires development, deployment, operation, and continuous upgrading of advanced modeling tools, software, and decision support systems (DSSs). Well known DSSs with application in the water sector include WEAP (SEI, 2018), Mike Basin (DHI, 2018), and MODSIM (Labadie, 2015) but much more are reportedly in use (e.g., Schardong and Simonovic, 2015; Srdjevic and Srdjevic, 2017). The data processing, analysis, and decision-making are important architectural components in all cases.
The main objective of the article is to propose an approach in combining those architectural components into one framework, as presented in Figure 2.

The next two subsections will briefly describe how processing of data can be performed (ACQUANET model) and which decision-making tool can be used (AHP and consensus convergence model) to evaluate scenarios, created after a detailed analysis of processed data.

\subsection{Network simulation-optimization model ACQUANET}

The network river basin computer model ACQUANET (LabSid, 2018) is a simpler version of MODSIM (Labadie, 2015), a well-known model/software for solving water allocation problems for multiyear periods. For known hydrological conditions, ACQUANET simulates the operation of reservoirs located at most upstream locations of a watershed and allocates water to downstream users according to initial storages in reservoirs, specified operating rule curves at reservoirs, and the given priority scheme in overall water distribution. A physical model, usually tree-structured, is automatically modified into a close capacitated network model with a set of nostorage nodes and numerous physical and (added) artificial links. As such, a new model is a closed network, which must 


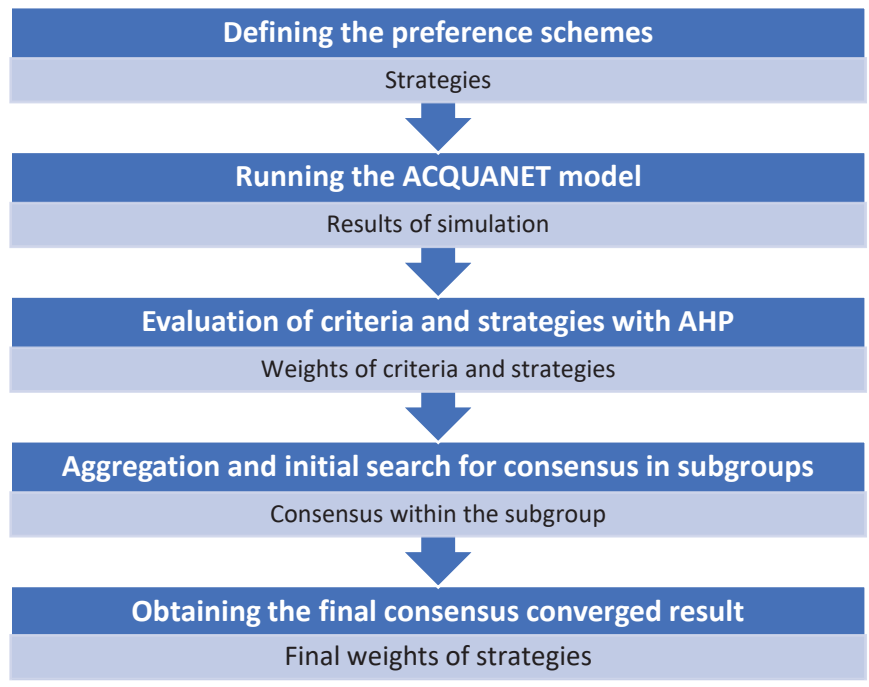

Figure 2. Methodology for integrating multi-criteria optimization with river basin simulation-optimization models

Abbildung 2. Methode zur Integration der Multi-Kriterien-Optimierung in Simulationsoptimierungsmodellen für Flußgebiete

be in balance during the given time step (which is defined as one calendar month). After input data are submitted to the model, the multiyear allocation problem is solved as a chain of consecutive monthly optimizations, transferring the necessary information from month-to-month to preserve continuous time flow. This way ACQUANET behaves as a network simulation-optimization model.

The model is strictly deterministic, with the base assumption that hydrological conditions are known for 1 month ahead. At the beginning of any month within the multiyear period, the model "knows" what inflows will happen during this month, the required storage levels to be met in reservoirs at the end of that month, the demands at all demand points, capacities at all links within a system (rivers, canals, pipelines, transfer waterways, etc.), and what are the priorities of all stated targets (reservoirs' storage levels and downstream demands).

The 1-month allocation problem is stated as network linear programming problem

$\min F=\sum_{i j} C_{i j} X_{i j}$, for all $i$ and $j$

subject to balance requirements at all nodes

$\sum_{j} X_{j i}-\sum_{j} X_{i j}=0$, for all $i$

and satisfaction of flow conditions at all links

$L_{i j} \leq X_{i j} \leq U_{i j}$, for all $i$ and $j$ where $i$ and $j$ are used to identify nodes in a network; $X_{i j}$ is the flow in the link $[i, j] ; C_{i j}$ is the unit cost of flow through the link $[i, j]$; and $L_{i j}$ and $U_{i j}$ are lower and upper limits on the flow through the link $[i, j]$.

The allocation problems (1)-(3) are solved for each month using given network parameters (inflows, demands, rule curves at reservoirs, capacities on links, demands' priorities scheme, etc.). The solver in ACQUANET is based on a Lagrangian overrelaxation algorithm, the network solver is written in Fortran, and its interface is created with Visual Basic. The ACQUANET model is selected for its simplicity, user friendliness, and possibilities to include into analysis irrigation and power generation-which suits to both case studies selected in Serbia and Portugal.

\subsection{The AHP method}

Among the methods for solving discrete optimization problems with more than one criterion and a number of alternatives, the AHP (Saaty, 1980) is one of the most used methods in both individual and group environments. This method efficiently handles one of the key issues in decision-making: eliciting judgments from the DM about the importance of a given set of decision elements regarding the overall goal, with criteria set introduced to locally prioritize (numerically) exposed judgments. If a problem can be structured hierarchically, then a ratio scale can serve as an effective tool to enable this hierarchy by performing pair-wise comparisons.

The core of the AHP lies in presenting the problem as a hierarchy (illustrated in Figure 3) and pairwise comparing the hierarchical elements using Saaty's 9-point scale (Saaty, 1980). In this way, the importance of one element over another is expressed with regards to the element in the higher level. The AHP creates the so-called local comparison matrices at all levels of a hierarchy and performs logical syntheses of their (local) priority vectors. The major feature of the AHP is that it can thus include in the same framework a variety of tangible and intangible goals, attributes, and other decision elements. In addition, it reduces complex decisions to a series of pairwise comparisons, implementing a structured, repeatable, and justifiable decision-making approach.

In the standard AHP, an eigenvector method is used to derive the weights from local matrices. After the local weights are calculated at all levels of the hierarchy, the synthesis consists of multiplying the criterion-specific weight of the 


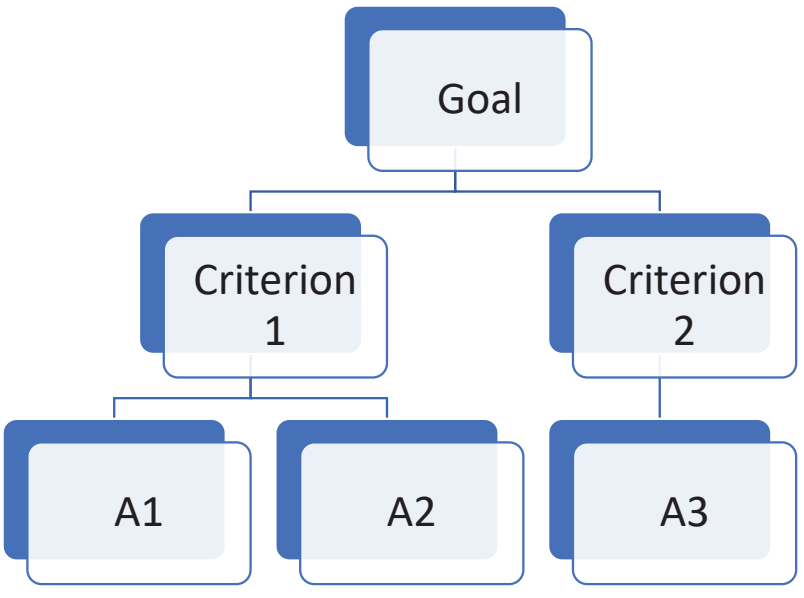

Figure 3. A hierarchy with a goal $(\mathrm{G})$, two criteria $\left(\mathrm{C}_{1}-\mathrm{C}_{2}\right)$, and three alternatives $\left(A_{1}-A_{3}\right)$

Abbildung 3. Die Hierarchie mit Ziel $(G)$, zwei Kriterien $\left(C_{1}-C_{2}\right)$ und drei Alternativen $\left(A_{1}-A_{3}\right)$

alternative by the corresponding criterion weight and summing up the results to obtain composite weights for the alternative with respect to the goal. This procedure is unique for all alternatives and all criteria.

The AHP is designed to support decision-making processes in both individual and group contexts. Its recent applications in water management that can be found, for example, in Blagojevic et al. (2016a, b), Amineh et al. (2017), Pluchinotta et al. (2018), Karlsson-Vinkhuyzen et al. (2018), and Srdjevic et al. (2018).

\subsection{Consensus in group decision-making}

In a group decision-making process, both consensus and consistency need to be pursued and sought after. A solution with a high level of consensus is desirable. Many researchers focus on how to define acceptable level of consensus and, in turn, how to achieve it (Moreno-Jimenez et al., 2008; Alonso et al., 2010; Bezerra et al., 2014; Blagoje- vic et al., 2016b; Brandt et al., 2017; Dong et al., 2017). Beside methods proposed in given literature, there are also other formal mathematical methods for reaching the consensus, such as consensus convergence modeling or central tendency methods (middle "value" is measured using the mean, median, or mode). One of the best-known formal models is the consensus convergence model (Lehrer and Wagner, 1981), where by repeatable mathematical procedure and through mutual respect, the DMs do not only achieve consensus on the issue under consideration but also agree on the overall relative weight of each member of the group.

This model is considered a suitable conflict resolution method in water management problems because its mathematical structure captures the typical situation of disagreement. Refusing to change one's opinion is equivalent, in mathematical terms, to assigning a null weight to other members and full weight to oneself (Hartmann et al., 2009). This situation is pure dogmatism, which is widely seen as unacceptable in modern decision-making.

\subsection{The consensus convergence model}

The central idea of the consensus convergence model is assigning the stakeholders' beliefs about the expertise of other epistemic stakeholders on the issue at hand (Hartmann et al., 2009). The weight of respect, $w_{i j}$, describes the regard stakeholder, $i$, has for the opinion or expertise of stakehold$\mathrm{er}, j$, and $\sum_{j=1}^{n} \omega_{i j}=1$ for the group of $n$ stakeholders.

Here we use an adapted version of the consensus convergence model presented in Regan et al. (2006). The procedure is based on the original model introduced by Lehrer and Wagner (1981), which uses the weights of respect assigned by each stakeholder, and modified model defined by Regan et al. (2006). The later model proposes using the weights of respect based on the strength of differences in criteria weights assigned by individuals in the group.

Table 1. Priorities of water use within management strategies for the period 2016-2025 as decision alternative for water allocation

Tabelle 1. Prioritäten der Wassernutzung innerhalb der Managementstrategien für die Periode 2016-2025 als Entscheidungsalternativen für die Wasserzuteilung

\begin{tabular}{lcccc}
\hline Water uses category & Strategy $\mathbf{1}$ & Strategy $\mathbf{2}$ & Strategy 3 & Strategy 4 \\
\hline IR - Irrigation & Top & Medium & Medium & Low \\
DR - Drainage & Medium & Top & Low & Medium \\
UW - Used waters & Low & Low & Top & Low \\
IS - Industrial supply & Medium & Medium & Medium & Top \\
OP - Other purposes & Negligible & Low & Low & Negligible \\
\hline
\end{tabular}


In this model, we can assume that initial criteria weights of $n$ stakeholders are $p_{1}^{0}, p_{2}^{0}, \ldots, p_{n}^{0}$ and a metric that calculates weights of respect is

$$
\omega_{i j}=\frac{1-\left|p_{i}^{0}-p_{j}^{0}\right|}{\sum_{j=1}^{n} 1-\left|p_{i}^{0}-p_{j}^{0}\right|}
$$

where $i$ refers to the individual who is assigning the weights, $j$ refers to the individual being assigned a weight, and $n$ is the number of group members.

The weights of respect are used to create $n \times n$ size matrix $W$

$$
W=\left[\begin{array}{cccc}
w_{1} & w_{1} & \ldots & w_{1 n} \\
w_{1} & w_{2} & \ldots & w_{1 n} \\
\ldots & \ldots & \ldots & \ldots \\
w_{n 1} & w_{n 2} & \ldots & w_{n}
\end{array}\right]
$$

If $P$ is a vector of initial criteria weights, consensual vector of criteria weights can be obtained by the iterative equation

$P_{c}=W P_{c-1}$

The procedure is repeated until the values of criteria weights in vectors $P_{c}$ and $P_{c-1}$ are equal within the tolerance error limit. Convergence is guaranteed if weights of respect are constant throughout the iteration process for each DM.

\section{Results and discussion}

\subsection{The stakeholders-participants in the decision- making process}

Participants in the decision-making process (stakeholders) are identified by responsible public water management authority (PWMA). A group of 23 invited individuals are briefed on main problems related to long-term planning and management of the hydro system, and particularly on a methodology applicable to resolving existing conflicts among water users. The final intent of a meeting was to gather parties around the same table and try to solve the common problem.

The participants are explained how to act in the decisionmaking session aimed to reach a consensus about a strategy aiming to achieve well-balanced system use and satisfaction of prescribed system purposes and users' expectations and also respecting the defined system capacity requirements and societal wider interests. A discussion helped to elaborate the most important decision-making issues and to define a global goal to identify the most desired longterm management strategy. Three criteria (identified as economic, social and ecological) and five purposes of the system (irrigation [IR], drainage [DR], used waters [UW]/ effluent from industry, industrial supply [IS], and other purposes [OP]) are adopted as evaluation filters that will apply to management strategies.

\subsection{Water allocation scenarios simulation and evaluation}

\section{Phase \# 1 (Defining the preference schemes)}

Four decision alternatives are representing possible management strategies for the 10-year period 2016-2025. Strategies are adopted after justification provided by the PWMA Vode Vojvodine and participants' notion of the global importance of different water uses. Preference schemes summarized in Table 1 are explained to the participants in detail, pointing the main concerns, possible conflicts in water allocation (both spatial and temporal), and so on.

Notice that from Table 1, one can easily see that in each strategy one of the purposes of the system is assumed to have dominant priority and that some purposes have tied priority.

Table 2. Weights of the strategies within one of the five subgroups

Tabelle 2. Gewichtung der Strategien innerhalb der fünf Untergruppen

\begin{tabular}{lcccc}
\hline & Strategy 1 & Strategy 2 & Strategy 3 & Strategy 4 \\
\hline Stakeholder1 & 0.393 & 0.239 & 0.175 & 0.193 \\
Stakeholder2 & 0.414 & 0.236 & 0.174 & 0.176 \\
Stakeholder3 & 0.411 & 0.249 & 0.204 & 0.136 \\
Stakeholder4 & 0.338 & 0.235 & 0.229 & 0.198 \\
Stakeholder5 & 0.334 & 0.219 & 0.226 & 0.220 \\
Consensus convergence weights & 0.366 & 0.233 & 0.212 & 0.190 \\
\hline
\end{tabular}




\section{Phase \#2 (Running the ACQUANET model)}

The model for regional hydro system Nadela is developed as shown in Figure 3. Simulations are performed for 10-year period, 2016-2025, based on statistically relevant historic data about precipitations because there are no other water inflows into the main canal. Because most upstream point in the model must be a reservoir, and because in this particular case, there is no such reservoir, an artificial reservoir with zero capacity is posted in the scheme to simulate intakes of water through the locker which connects Nadela canal with the Danube-Tusza-Danube (DTD) canal system; cf. the triangle at Figure 4 at the top-north point of the scheme.

System is supplied by $5 \mathrm{~m}^{3} \mathrm{~s}^{-1}$ of water from DTD, and the maximum capacity is $20 \mathrm{~m}^{3} \mathrm{~s}^{-1}$ (for the situations when system is used for the drainage). Note that environmental flow needs are set to $0.5 \mathrm{~m}^{3} \mathrm{~s}^{-1}$ within the model. Also, although very important, note that water quality issues are out of the scope of the article as it cannot be modeled with ACQAUNET and requires additional modeling.

Phase \#3 (Individual evaluation of criteria and other decision elements with AHP)

A session started with a 15-min brainstorming during which participants from PWMA Vode Vojvodine and the Provincial Secretariat for Agriculture, Forestry and Water Management of the AP Vojvodina found the compromise about relative mutual importance of the three selected criteria. The resulting weights of criteria are derived by the eigenvector prioritization of the AHP as follows: economic criterion of 0.674 , social criterion of 0.226 , and ecological criterion of 0.101 . This occurred following the part of a session where all participants expressed their semantic preferences while performing pairwise comparisons of elements at the third and fourth level of a hierarchy by judging elements in one level regarding elements in higher level. Each individual filled in 8 comparison matrices: three $5 \times 5$ matrices for comparisons of water uses against three criteria, and five $4 \times 4$ matrices for comparisons of four offered strategies against five water uses. The resulting local weights of water uses were computed in turn and the AHP calculated the final weights of strategies for each participant.

Table 4. The final weights of alternative water allocation strategies Tabelle 4. Die entgültige Gewichtugn der alternativen Wasserzuteilungsstrategien

\begin{tabular}{lcccc}
\hline Aggregation method & Strategy 1 & Strategy 2 & Strategy 3 & Strategy 4 \\
\hline Consensus convergence & $0.167(4)$ & $0.204(3)$ & $0.341(1)$ & $0.285(2)$ \\
\hline
\end{tabular}

Table 3. Subgroups consensus weights for strategies Tabelle 3. Konsensgewichte der Untergruppen für die Strategien

\begin{tabular}{lcccc}
\hline Strategy/Subgroups & Strategy 1 & Strategy 2 & Strategy 3 & Strategy 4 \\
\hline Subgroup 1 & 0.366 & 0.233 & 0.212 & 0.190 \\
Subgroup 2 & 0.132 & 0.222 & 0.421 & 0.225 \\
Subgroup 3 & 0.187 & 0.304 & 0.310 & 0.199 \\
Subgroup 4 & 0.128 & 0.163 & 0.304 & 0.405 \\
Subgroup 5 & 0.156 & 0.161 & 0.386 & 0.297 \\
\hline
\end{tabular}

Phase \#4 (Aggregation and initial search for consensus in subgroups)

Stakeholders were grouped into five subgroups according to their affiliation, responsibility, and present professional (or political) function related to water resources and/or regional system. Subgroups are formed as follows: Subgroup 1: Irrigation (5 stakeholders); Subgroup 2: Used waters (3); Subgroup 3: State/public interest (7); Subgroup 4: Industry (4); and Subgroup 5: Local authorities (4). For illustration purposes, individually derived weights of the strategies within the Subgroup 1 by AHP are presented in Table 2 .

To obtain consensus weights of strategies within each subgroup, weights of respect of each stakeholder within the same subgroup are calculated for each strategy and placed in "weights of respect matrix" W. For example, for Strategy 1 within Subgroup 1, $W^{s 1}$ is provided by using the vector of initial Strategy 1 weights and applying equation 5:

$$
W^{s 1}=\left[\begin{array}{lllll}
0.206 & 0.202 & 0.203 & 0.195 & 0.194 \\
0.203 & 0.207 & 0.207 & 0.192 & 0.191 \\
0.203 & 0.206 & 0.207 & 0.192 & 0.191 \\
0.197 & 0.193 & 0.193 & 0.209 & 0.208 \\
0.197 & 0.192 & 0.193 & 0.208 & 0.209
\end{array}\right]
$$

By applying equation 6 , consensual convergence weight of 0.366 for Strategy 1 is obtained, and this value can easily be compared with individual values in Table 3 . The last row of this table contains consensus convergence weights for all strategies within Subgroup 1. Using the same method, consensus weights for all other subgroups are computed and presented in Table 3.

Phase \#5 (Obtaining the final consensus converged result) Finally, subgroup decisions from Table 3 are aggregated into the group decision by reaching the consensus between the subgroups. Steps in applying consensus convergence model are (1) calculate weights of respect among the subgroups for each strategy; (2) place weights in an appropriate matrix, and (3) compute consensus convergence weight of each strategy. 

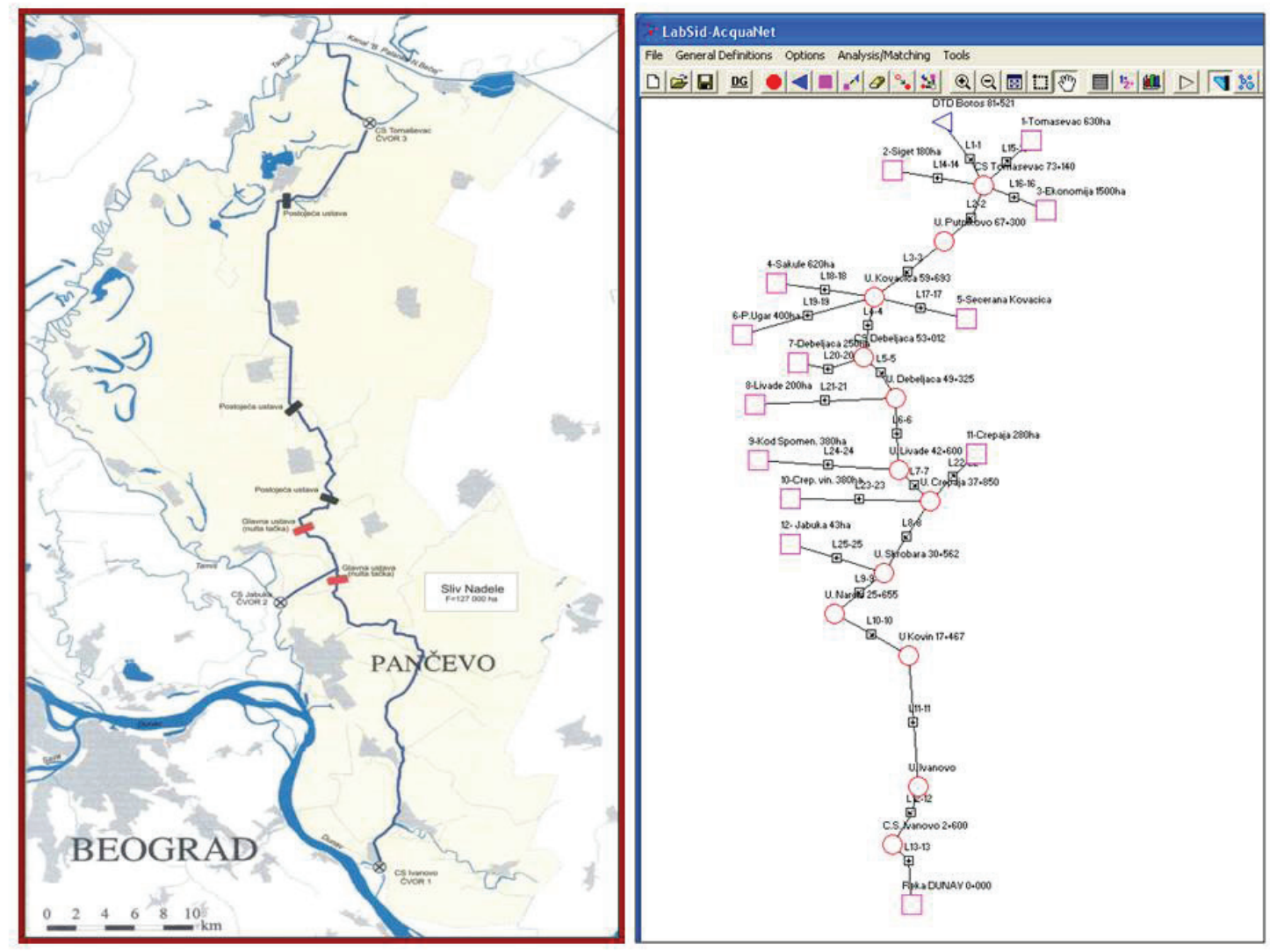

Figure 4. ACQUANET model for regional Nadela hydro system Abbildung 4. ACQUANET-Modell für das regionale Wassersystem Nadela

Note that in Phase \#4, weights of respect were calculated between the participants within one subgroup at a time, whereas in this phase, weights represent respect of given subgroup toward the other subgroups.

The final result (consensus weights) is presented in Table 4 . Strategy 3 is identified as the best management alternative and Strategy 4 as the second best. Strategies 1 and 2 changed ranks in two aggregation schemes.

It is interesting to note that the top-ranked Strategy 3 reflects stakeholders' opinion that the most important water use category in the Nadela watershed is "Used water," rather than "Irrigation" and "Industry supply." In reality, downstream sector of the Nadela canal is frequently polluted by used waters from the sugar industry (Kovacica) and milk factory (Pancevo) and this skewed the decision-making process toward the scenarios ranking presented in Table 4.

The final part of a session and discussion between stakeholders about the results of applied methodology showed that significant majority of participants (stakeholders) was satisfied with the final ranking of possible water allocation strategies and especially with the best strategy identified.

\section{Conclusions}

This article addresses the issue of water management in the modern world, characterized by conflict between different water users. Each watershed has its own specific problems, but what is common is that conflict could be better managed if stakeholders participate in the whole process of decision-making: defining problem, defining priorities in water management, and evaluating the results of simulating those strategies. Developing the methodology to combine all these aspects into one framework was the objective of the bilateral project "Multi-criteria decision making/analytic hierarchy process applications in solving problems of priority water allocation for agricultural users" and of the article as well. Proposed methodology consists of five phases: de- 
fining the preference schemes, running the ACQUANET model, evaluating criteria and strategies with AHP, aggregation and initial search for consensus in subgroups, and obtaining the final consensus converged result.

This approach is tested on the water allocation problem in the Nadela watershed in Vojvodina Province in Serbia. Twenty-three stakeholders participated in defining allocation priority schemes as a set of four scenarios considering local, regional, and state-wide conditions, with focus on preferences of local stakeholders. After multiyear simulation of management strategies, stakeholders evaluated strategies with respect to economic, social, and ecological criteria. Consensus of 23 stakeholders, divided into 5 subgroups, was then reached by a formal mathematical model. When the results obtained have been presented to participants, they have agreed by acclamation that the best strategy is really the one identified by the methodology and that it is likely to have it implemented.

A similar approach will be applied for the area near Bragança in northeast Portugal (Sabor watershed), with the inclusion of stakeholders from the tourism and energy production sector, as sectors very important to the area. At a later stage of the bilateral project, search for best solutions will be combined with the assessment of sustainability criteria and related indicators, following ideas presented in previous research (Sandoval-Solis et al., 2011; Srdjevic and Srdjevic, 2017).

Authors are also aware of the importance of the water quality issues, but it was out of the scope of this article. Further research should thus explore and model the influence of selected allocation strategy on water quality in selected watersheds.

\section{Acknowledgments}

This work was supported in part by the Ministry of Education, Science and Technological Development of Serbia under the grant 174003 (2011-2018) - Theory and application of Analytic hierarchy process (AHP) in multicriteria decision-making under conditions of risk and uncertainty (individual and group context).

The authors would also like to acknowledge the financial support received from the Serbian Ministry of Education, Science and Technological Development under Grant No. 451-03-1924/2016-09/38, and the FCT/Portugal support via bilateral project grant 441.00 SERBIA.

\section{References}

Alonso, S., Herrera-Viedma, E. and F. Herrera (2010): A web based consensus support system for group decision making problems and incomplete preferences. Journal of Information Sciences 180, 4477-4495.

Amineh Z.B.A., Hashemian S.J. and A. Magholi (2017): Integrating spatial multi criteria decision making (SMCDM) with Geographic Information Systems (GIS) for delineation of the most suitable areas for aquifer storage and recovery (ASR). Journal of Hydrology 551, 577-595.

Bezerra, F., Melo, P. and J.P. Costa (2014): Visual and interactive comparative analysis of individual opinions: a group decision support tool. Group Decision and Negotiation 23, 101-125.

Blagojevic, B., Srdjevic B., Srdjevic, Z. and T. Zoranovic (2016): Heuristic aggregation of individual judgments in AHP group decision making using simulated annealing algorithm. Information Sciences 330, 260-273.

Blagojevic, B., Srdjevic, Z., Bezdan, A. and B. Srdjevic (2016): Group decision making in land evaluation for irrigation: A Case study from Serbia. Journal of Hydroinformatics 18, 579-598.

Brandt, P., Kvakić, M., Butterbach-Bahl, K. and M.C. Rufino (2017): How to target climate-smart agriculture? Concept and application of the consensus-driven decision support framework "targetCSA". Agricultural Systems 151, 234-245.

DHI - Danish Hydraulic Institute (2004): MIKE-BASIN environment for integrated water resources management and planning. https://www.mikepoweredbydhi. com/products/mike-hydro-basin. Accessed on 6 May 2019.

Dong, Q., Zhü, K. and O. Cooper (2017): Gaining consensus in a moderated group: A model with a twofold feedback mechanism. Expert Systems with Applications 71, 87-97.

Hartmann, S., Martini, C. and J. Sprenger (2009): Consensual decision-making among epistemic peers. Episteme 6, 110-129.

Karlsson-Vinkhuyzen, S., Boelee, E., Cools, J., van Hoof, L., Hospes, O., Kok, M., Peerlings, J., van Tatenhove, J., Termeer, C.J.A.M. and I.J. Visseren-Hamakers (2018): Identifying barriers and levers of biodiversity mainstreaming in four cases of transnational governance of land and water. Environmental Science \& Policy 85, 132-140. 
Labadie, J.W. (2015): MODSIM - Decision support system for integrated river basin management. Documentation and User Manual. Colorado State University, Fort Collins, USA.

LabSid (2018): AcquaNet - River basin simulation model. User Manual. University of San Paulo, Brazil. http:// www.labsid.eng.br/software.aspx?id=14. Accessed on 13 May 2019.

Lehrer K. and C. Wagner (1981): Rational Consensus in Science and Society. Reidel, Dordrecht.

Moreno-Jimenez, J.M., Aguaron, J. and M.T. Escobar (2008): The Core of Consistency in AHP-Group Decision Making. Group Decision Negotiations, 17, 249-265.

Pluchinotta, I., Pagano, A., Giordano, R. and A. Tsoukiàs (2018): A system dynamics model for supporting decision-makers in irrigation water management. Journal of Environmental Management 223, 815-824.

Regan, H.M., Colyvan, M. and L. Markovchick-Nicholls (2006): A formal model for consensus and negotiation in environmental management. Journal of Environmental Management 80, 167-176.

Saaty, T. (1980): Analytic Hierarchy Process. McGraw Hill, New York, USA.

Sandoval-Solis, S., McKinney, D.C. and D.P. Loucks (2011): Sustainability index for water resources planning and management. Water Resources Planning and Management 2011, 381-390.
Schardong, A. and S.P. Simonovic (2015): Coupled selfadaptive multiobjective differential evolution and network flow algorithm approach for optimal reservoir operation. Journal of Water Resources Planning and Management 141, 04015015.

Srdjevic, B., Melo, P., Srdjevic, Z., Jorge, L. and T. Zoranovic (2019): Water allocation for agricultural users based on multi criteria analysis and use of decision-making tools. In: The Proceedings of the $47^{\text {th }}$ International Symposium "Actual tasks on Agricultural Engineering". 5-7 March 2019, Opatija, Croatia, pp. 117-126.

Srdjevic, Z. and B. Srdjevic (2019): Multistakeholder conflicts and resolutions in water resources management. In: The Proceedings of the $47^{\text {th }}$ International Symposium "Actual tasks on agricultural engineering", Opatija, Croatia, 5-7 March 2019, 127-134.

Srdjevic, B., Srdjevic, Z. and M. Lakicevic (2018): Validating the importance of criteria for assessing climate change scenarios. Water and Climate Change 9, 570-583.

Srđević, Z. and B. Srđević (2017): An extension of the sustainability index definition in water resources planning and management. Water Resources Management 31, $1695-1712$.

SEI - Stockholm Environment Institute (2018): WEAP Water evaluation and planning system. https://www. weap21.org/. Accessed on 6 May 2019. 